\title{
Entre patrimonialisation et ouverture au tourisme : un équilibre en question(s) dans le lagon du Grand Cul-de-Sac Marin en Guadeloupe
}

Between Heritage Making and Tourism Development: Looking for a Good Balance in the Lagoon of Grand Cul-de-Sac Marin in Guadeloupe

Entre la patrimonialización y el desarollo turístico : buscando un equilibrio en la laguna del Grand Cul-de-Sac Marin en Guadalupe

Vincent Andreu-Boussut, Caroline Rufin-Soler et Céline Chadenas

\section{OpenEdition}

Journals

Édition électronique

URL : https://journals.openedition.org/ideas/3834

DOI : $10.4000 /$ ideas.3834

ISSN : $1950-5701$

Éditeur

Institut des Amériques

Référence électronique

Vincent Andreu-Boussut, Caroline Rufin-Soler et Céline Chadenas, « Entre patrimonialisation et ouverture au tourisme : un équilibre en question(s) dans le lagon du Grand Cul-de-Sac Marin en Guadeloupe »I IdeAs [En ligne], 12 | 2018, mis en ligne le 21 novembre 2018, consulté le 18 octobre 2022. URL : http://journals.openedition.org/ideas/3834; DOI : https://doi.org/10.4000/ideas.3834

Ce document a été généré automatiquement le 18 octobre 2022

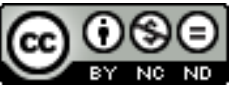

Creative Commons - Attribution - Pas d'Utilisation Commerciale - Pas de Modification 4.0 International - CC BY-NC-ND 4.0

https://creativecommons.org/licenses/by-nc-nd/4.0/ 


\section{Entre patrimonialisation et ouverture au tourisme : un équilibre en question(s) dans le lagon du Grand Cul-de-Sac Marin en Guadeloupe}

Between Heritage Making and Tourism Development: Looking for a Good Balance in the Lagoon of Grand Cul-de-Sac Marin in Guadeloupe

Entre la patrimonialización y el desarollo turístico : buscando un equilibrio en la laguna del Grand Cul-de-Sac Marin en Guadalupe

Vincent Andreu-Boussut, Caroline Rufin-Soler et Céline Chadenas

Cet article est issu du programme de recherche PRESSILES « PRESSions touristiques sur les ILEts du Parc national de Guadeloupe " financé par le Parc national de Guadeloupe de 2016 à 2018 en partenariat avec le Conservatoire du Littoral. Des remerciements doivent être adressés ici à Anthony Tricq et à Léa Henry pour leur collaboration dans les enquêtes, à Nicolas Rollo de l'Université de Nantes pour le traitement des données statistiques, à Philippe Chagnon et à Simon Charrier pour le traitement des données spatiales et les réalisations cartographiques, et à Xavier Delloue du Parc national de Guadeloupe pour son soutien.

\section{Introduction}

1 La recherche d'un équilibre entre développement touristique et protection de la nature est loin d'être une question nouvelle. Pour les gestionnaires d'espaces naturels, la conciliation entre objectifs de conservation patrimoniale et maintien ou croissance de la fréquentation touristique est depuis une vingtaine d'années un impératif stratégique, poussé par la double nécessité de générer des bénéfices économiques et de consolider la légitimité et l'efficacité des projets de protection. Cette question opérationnelle, à la 
fois technique et politique, a ainsi fait l'objet d'un important investissement scientifique et les guides méthodologiques à disposition des professionnels de la conservation s'enchaînent depuis le début des années 2000 (Eagles et al., 2002, Foxlee, 2007, Leung et al., 2015). Même si cette littérature grise explore les meilleurs résultats en la matière (Andreu-Boussut et al., 2016), nombreux sont les exemples de surfréquentation (Meur-Férec, 2007) et d'accaparement des ressources naturelles pour le tourisme au détriment de la population locale (Grenier, 2000). La gestion des impacts écologiques, sociaux et économiques de la fréquentation touristique des espaces protégés reste donc une question d'actualité, sans cesse remodelée au gré des contextes évolutifs des usages de la nature.

2 En Guadeloupe, le développement touristique est resté focalisé sur les usages balnéaires jusqu'aux années 1990, à l'origine d'une concentration des hébergements et des activités sur la Grande-Terre le long de la Riviera du Levant entre Le Gosier et SaintFrançois. La création d'un Parc national en 1989 sur la Basse-Terre (fig. 1) inaugure une période favorable à une plus grande durabilité touristique (Grandbois, 2004, Breton, 2004). La recherche d'un équilibre entre développement et protection se pose alors, à la fois parce que les sites emblématiques du Parc national connaissent une croissance importante de leur fréquentation ${ }^{1}$, mais aussi parce que les stratégies de développement touristique de l'archipel font à partir des années 2000 le choix d'un tournant en faveur de l'écotourisme et du tourisme de nature (Bouin, 2004, Olier, 2004, Dehoorne, 2011). Au Nord de la Guadeloupe, la baie du Grand Cul-de-Sac Marin est restée un temps à l'écart de la dynamique touristique guadeloupéenne. Fermé par le plus grand récif barrière des Petites Antilles, ce lagon de plus de 11000 hectares constitue un espace de ressources marines à l'échelle locale qui fait depuis trente ans l'objet d'un processus concomitant de mise en tourisme et de mise en patrimoine. D'un côté, ses îlets sont devenus des destinations privilégiées de détente pour les Guadeloupéens avant de s'ouvrir au tourisme comme destinations d'excursions «tout compris " pour la baignade et pour la découverte des écosystèmes marins et de la mangrove (Desvergnes et Vincent, 2011). Ce faisant, certains îlets sont devenus les symboles du paysage touristique caribéen. D'un autre côté, une patrimonialisation est engagée dès 1987 par la création d'une Réserve naturelle sur une partie de ses îlets et de ses mangroves, avant d'être spatialement amplifiée à l'intégralité de la baie en 2009 par l'extension du nouveau périmètre du Parc national (fig. 1).

Fig. 1 : Les espaces de la protection de la nature à l'échelle de l'archipel de la Guadeloupe. 


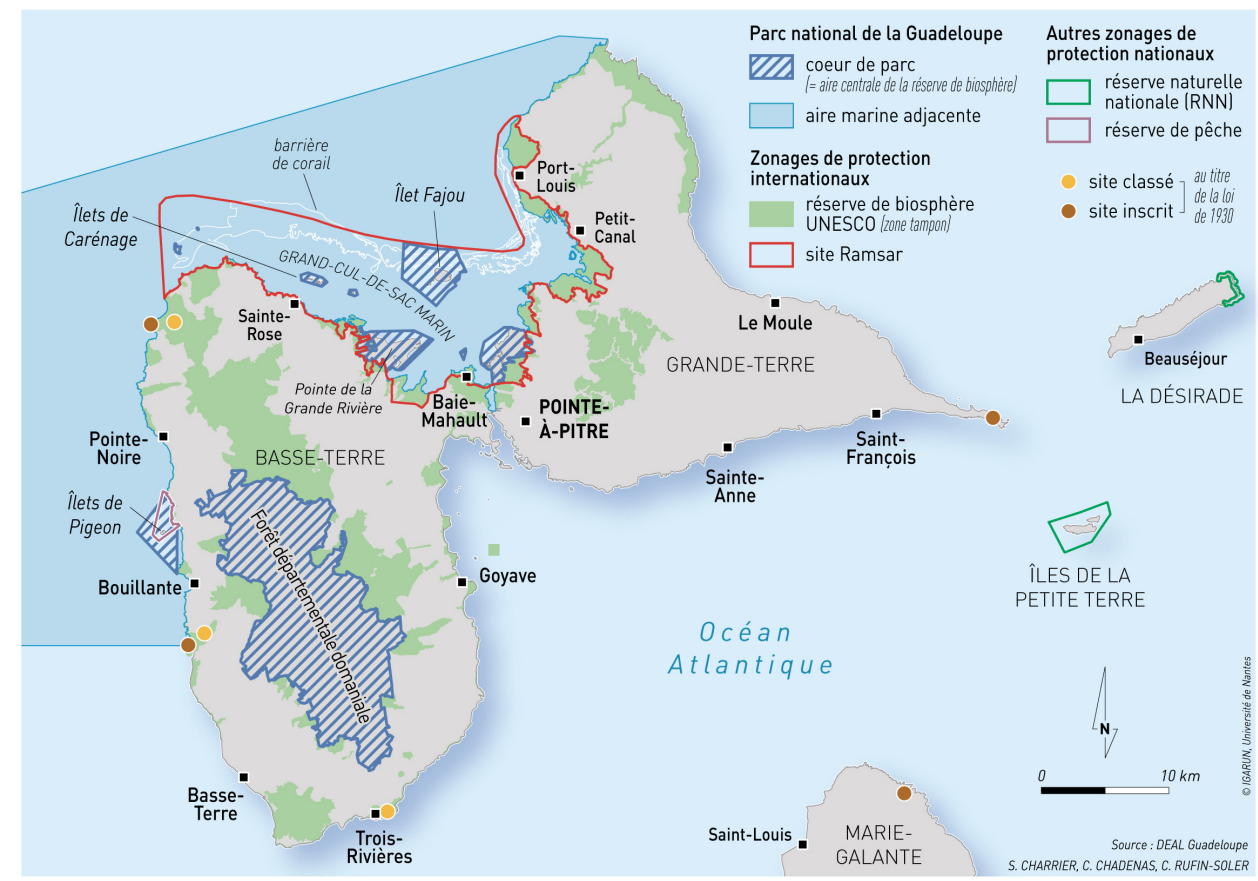

3 Trois questions émergent de cette situation. Quels sont les rouages géo-historiques de ce double processus de mise en patrimoine et en tourisme qui expliquent la construction du Grand Cul-de-Sac Marin comme haut-lieu touristique et patrimonial ? Dans un contexte de changement climatique, quels sont les enjeux d'achoppement et de conciliation entre le projet patrimonial porté par le Parc national et la continuité des activités touristiques développées par les collectivités locales et surtout par des prestataires privés? Si Nicole Olier notait en 2004 que «les communautés locales et leurs élus ne partagent pas la même perception quant à la mise en valeur environnementale et vivent parfois l'institution que constitue le Parc comme un frein réglementaire [...] au développement local» (Olier, 2004:408), comment les visions de l'ensemble des parties prenantes de la gestion du Grand Cul-de-Sac Marin ont-elles évolué depuis 15 ans? Autrement dit, la poursuite de la patrimonialisation institutionnelle s'est-elle accompagnée d'une appropriation des usagers et de l'ensemble des acteurs de la baie?

4 L'analyse présentée ici est issue d'une enquête à grande échelle par entretiens semidirectifs réalisés entre mai 2017 et mars 2018. Les guides d'entretien ont été coconstruits avec le Parc national de Guadeloupe et le Conservatoire du Littoral et ont été adaptés en fonction des deux groupes d'acteurs locaux ciblés, décomposés eux-mêmes en trois catégories distinctes. Ainsi, 63 entretiens ont pu être collectés auprès des usagers, dont 20 entretiens auprès d'habitants, 21 auprès de visiteurs touristiques et 22 auprès de visiteurs d'origine guadeloupéenne résidant en France métropolitaine. En parallèle, 36 entretiens ont été réalisés auprès d'acteurs territoriaux dont 13 institutions et acteurs publics (collectivités locales, services de l'État...), 7 associations et 16 professionnels du tourisme (essentiellement les prestataires de la baie). Malgré quelques variations, les entretiens ont suivi un déroulé identique: identité ou structure, pratiques spatiales, connaissances et représentations de la protection, perceptions de l'état écologique et de la fréquentation touristique et relations avec le Parc national et besoins en aménagements. La réalisation d'une carte mentale dessinée 
sur un fond de carte a par ailleurs permis de reconstituer les itinéraires empruntés dans la baie (ports de départ, trajets, destinations). Tous les entretiens ont fait l'objet d'une retranscription littérale selon le principe de fidélité (Romelaer, 2005) et d'une analyse multithématique.

\section{1- Un haut-lieu touristique et patrimonial : une patrimonialisation obligée d'intégrer les usages touristiques}

5 Les étapes de la mise en tourisme et en patrimoine expliquent l'invention du Grand Culde-Sac Marin comme haut-lieu touristique et patrimonial et démontrent que la patrimonialisation ne s'est jamais totalement accompagnée d'un projet préservationniste.

\section{Un espace nourricier doublé d'une destination de loisirs familiaux}

6 Le Grand Cul-de-Sac Marin connaît un essor remarquable des activités de pêche dès la fin du $\mathrm{XIX}^{\mathrm{e}}$ siècle poussé par la croissance démographique qui suit l'abolition de l'esclavage en 1848. La communauté des marins pêcheurs y développe une très grande diversité de métiers exploitant l'ensemble des espèces disponibles (crustacés, mollusques, poissons). A partir des années 1960, la pêche s'intensifie, tout comme la chasse et la cueillette, à la fois par la croissance de l'effort de pêche mais aussi par la « démocratisation de l'exploitation de la baie » (Buttifant et al., 2008), car de nombreux pêcheurs de loisirs viennent progressivement cohabiter avec les pêcheurs professionnels. Pratiques de subsistance, cette pêche et cette cueillette qui privilégient les crabes, les palourdes, les chaubettes et les lambis (en plongée) sont aussi ludiques :

$\mathrm{Au}$ mois de mai quand on était jeunes, avec tous les copains, on allait pêcher les crabes à barbe, dans la saison de Pâques [...]. Avec des pripris, on fabriquait des pripris, c'est des bouts de bois qui flottaient bien, on les traversait avec des planches [...], on s'amusait, c'était ce qu'on avait (entretien avec un habitant de Sainte-Rose, le 27 février 2018).

7 Avec l'émergence des loisirs pour les populations locales dans les années 1980, le lagon devient un espace de cohabitation entre le monde de la pêche et celui des loisirs. Les îlets, jusqu'alors utilisés par les pêcheurs comme bases de travail et de repli, deviennent des destinations le temps du week-end et particulièrement au moment des festivités de Pâques à l'occasion de grands repas familiaux où le crabe, pêché sur place, est souvent à l'honneur :

Nous, on a eu l'occasion d'aller sur les îlets, par exemple à Caret, passer la journée là-bas, camper à Caret, partir pour 3 ou 4 jours, mais Caret qui était dans le temps vraiment une belle île, il y avait des arbres, il y avait des cocotiers, et même des carbets de pêcheurs. Oui jusqu'à encore une vingtaine d'années, je dirais. On partait $\mathrm{du}$ bateau à Sainte-Rose avec la famille, et on arrivait à Caret, quelquefois c'était la Biche, un îlet qui est plus proche de Sainte-Rose [...].On allait à Fajou aussi (entretien avec des habitants de Morne-à-l'eau, le 26 février 2018).

Dans les années 1990, les trois quarts des visiteurs des îlets (Caret ou Fajou) sont donc des visiteurs locaux (Parc national de Guadeloupe, 1997, Olier, 2004) et l'attachement 
des habitants à l'espace nourricier et de loisirs que représente le Grand Cul-de-Sac Marin reste très fort encore aujourd'hui.

\section{La transformation du lagon en haut-lieu patrimonial par l'extension du Parc national en mer}

Concomitante de la mise en loisirs et en tourisme, le lagon fait l'objet d'une mise en patrimoine qu'il est possible de restituer en trois phases distinctes (fig. 2).

La première phase d'initiation de la patrimonialisation du Grand Cul-de-Sac Marin profite de la dynamique de protection entamée dès 1970 dans la Basse-Terre avec la création d'un Parc naturel départemental. Ce cadre institutionnel permet la préfiguration d'un Parc national qui est créé en 1989 dans un but de conservation de milieux tropicaux. La protection centrée sur le massif forestier de la Basse-Terre initie un mouvement en faveur de la protection du patrimoine naturel à l'échelle du territoire guadeloupéen. C'est à ce moment charnière, en 1987, que la Réserve naturelle du Grand Cul-de-Sac Marin est créée sur un périmètre morcelé (fig. 2) de 1622 ha de mangrove et de forêt et de 2115 ha de mer territoriale reconnue pour sa richesse en gorgones et en espèces coralliennes ${ }^{2}$. Le jeune Parc national devient gestionnaire de la réserve dès 1990 et dispose dès lors de prérogatives sur le lagon.

Fig. 2 : Frise chronologique et spatiale de la patrimonialisation du Grand Cul-de-Sac Marin.

Source : S. Charrier, C. Chadenas, C. Rufin-Soler, V. Andreu-Boussut, S. Angonnet

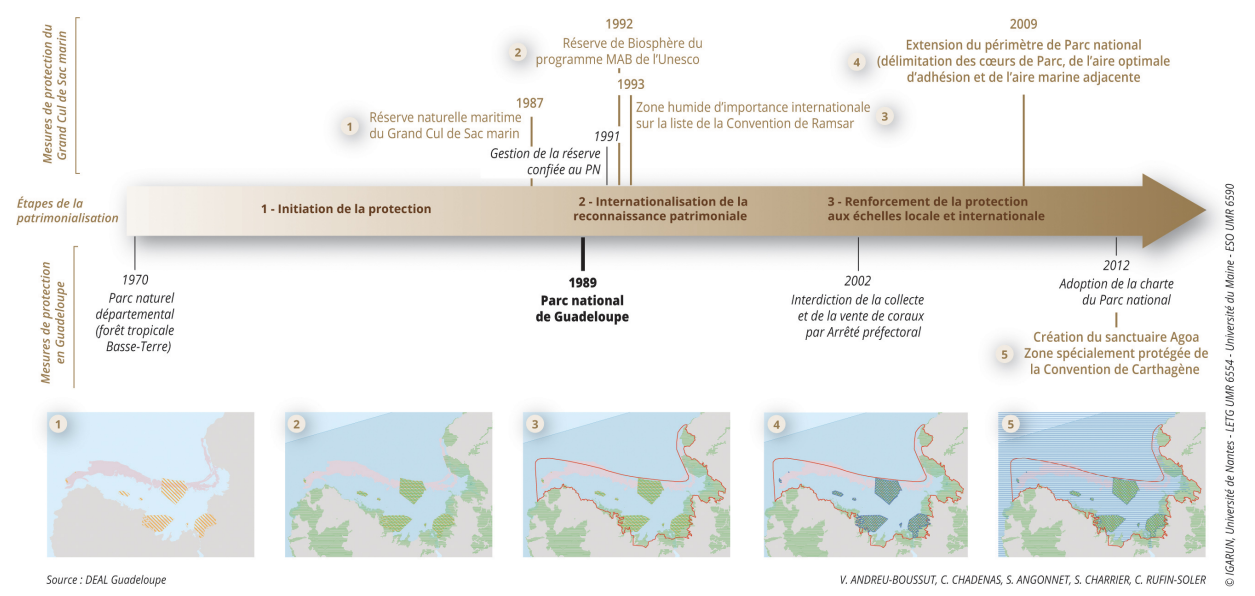

11 Une seconde phase d'internationalisation de la reconnaissance patrimoniale est ensuite engagée dès le début des années 1990. Elle s'appuie, d'une part, sur la création d'une Réserve de Biosphère du Réseau Man and Biosphere (MAB) de l'Unesco en 1992, et, d'autre part, sur l'inscription de la baie comme Zone humide d'importance internationale au titre de la Convention de Ramsar en 1993. La Réserve de Biosphère joue ici un rôle triple. Son zonage distingue une zone centrale de 22144 ha intégralement calée sur les périmètres du Parc national et de la Réserve naturelle, ce qui vient conforter ces territoires comme hauts-lieux de la protection de la biodiversité. Ensuite, la délimitation d'une zone tampon de 30394 ha et d'une zone de transition de 66416 ha, permet de construire un projet de protection plus large que les périmètres initiaux et vient s'étendre en mer. Le cadre territorial de la gestion de la biodiversité, confiée au Parc national, correspond désormais à la majeure partie de 
l'archipel guadeloupéen, débordant et s'affranchissant des périmètres de protection réglementaires. Un plan de gestion est mis en œuvre à partir de 1998 et le périmètre est même revu à la hausse en 2014. Enfin, la création de la Réserve de Biosphère donne une valeur internationale à la biodiversité et reconnaît aussi une valeur culturelle à l'ensemble du territoire (sites amérindiens, paysages agricoles de l'esclavage) tout comme elle privilégie dans les zones tampon et de transition des interactions nature/ culture favorables à la qualité des paysages et à la biodiversité. L'inscription de la baie en 1993 au titre de la Convention de Ramsar participe aussi du renforcement de la valeur internationale du patrimoine naturel du lagon mais offre surtout un cadre de gestion sur l'intégralité de son extension spatiale (fig. 2). Le suivi du site Ramsar est encore confié au Parc national, pour lequel l'inscription de la baie fournit un cadre de gestion cohérent car non morcelé tout en renforçant la stratégie d'utilisation rationnelle des ressources naturelles.

Dès la fin des années 1990, le Parc national est l'acteur patrimonial central dont la légitimité d'intervention déborde très largement son périmètre réglementaire strict. C'est donc dans une certaine continuité que s'enclenche la troisième phase de la patrimonialisation du Grand Cul-de-Sac Marin par le renforcement de la protection à l'échelle locale, et par l'intégration à un territoire plus vaste de protection des mammifères marins. Suite à la réforme du statut des Parcs nationaux français en 2006, le périmètre, le zonage et le fonctionnement du Parc national de Guadeloupe sont modifiés en 2009. Finalement, la mise en œuvre de la Réserve de Biosphère et du site Ramsar ont inauguré la préfiguration de l'extension du Parc sur le lagon. Dix espaces de "Cœurs de Parc » représentatifs de la quasi-totalité des écosystèmes de la Caraïbe sont identifiés dont huit correspondent à la Réserve naturelle originelle également zone centrale de la Réserve de Biosphère. Les deux autres cœurs sont constitués des îlets Pigeon et surtout de la zone centrale du précédent Parc national. Une aire d'adhésion non définitive est délimitée sur 21 des 32 communes qui composent le territoire guadeloupéen et l'aire optimale d'adhésion. Un troisième zonage, l'Aire maritime adjacente, complète le dispositif en couvrant toute la baie de la pointe de la Grande Vigie à l'est jusqu'au nord de Basse-Terre ainsi que toutes les eaux territoriales à l'ouest de la Guadeloupe. Suite à l'extension de ce périmètre, la charte de territoire est adoptée début 2012 et la Réserve naturelle est déclassée dès 2009. Ainsi, la refonte du Parc national s'inscrit pleinement dans la relance de la politique ultramarine qui privilégie depuis 2008 la construction d'aires marines protégées qui sont davantage des espaces de mise en valeur des ressources que des projets conservationnistes (Féral, 2011). Toute la baie est également intégrée au Sanctuaire Agoa créé en 2010 pour la protection des mammifères marins. Son emprise correspond à l'ensemble de la Zone économique exclusive des Antilles françaises, qui acquiert ainsi le statut d'aire marine protégée internationale au titre du protocole SPAW (Specially Protected Areas and Wildlife) de la Convention de Carthagène.

Fig. 3 : Périmètres de protection de la nature et des paysages à l'échelle du Grand Cul-de-Sac Marin.

Source : S. Charrier, C. Chadenas, C. Rufin-Soler, V. Andreu-Boussut 


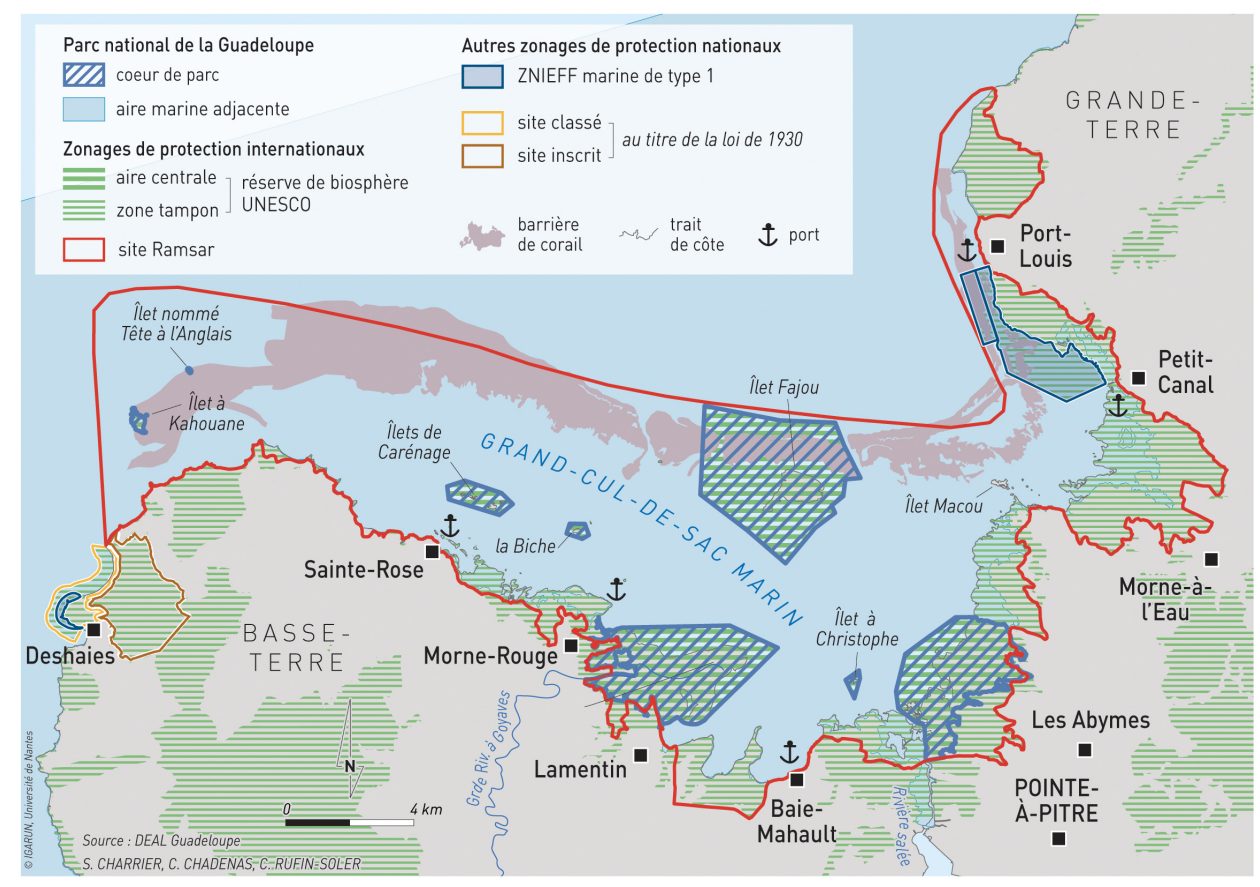

13 L'ensemble du processus de mise en protection (fig. 3) laisse la baie largement ouverte aux usages touristiques, tandis que les Cœurs du Parc calqués intégralement sur le périmètre initial de la Réserve naturelle historique sont soumis à réglementation.

\section{Le développement des usages touristiques à partir des années 1990 : une destination d'excursions en cours de diversification aux sports de nature}

14 Si la Guadeloupe devient une destination touristique à partir des années 1960, c'est à partir du début des années 1990 que la croissance des arrivées de touristes est la plus forte (fig. 4). 1998 marque d'ailleurs un pic avec 693000 arrivées qui précède une longue période de crise dont le paroxysme est atteint en 2009 à l'occasion de la crise sociale qui affecte l'archipel et s'accompagne d'importantes grèves.

Fig. 4 : Evolution de la fréquentation touristique de la Guadeloupe de 1986 à 2017. Le nombre de touristes comprend les touristes de séjour et les croisiéristes.

Sources : Bouin, 2004, Camus et Neiss, 2014, INSEE, Observatoire régional du Tourisme.

V. Andreu-Boussut 


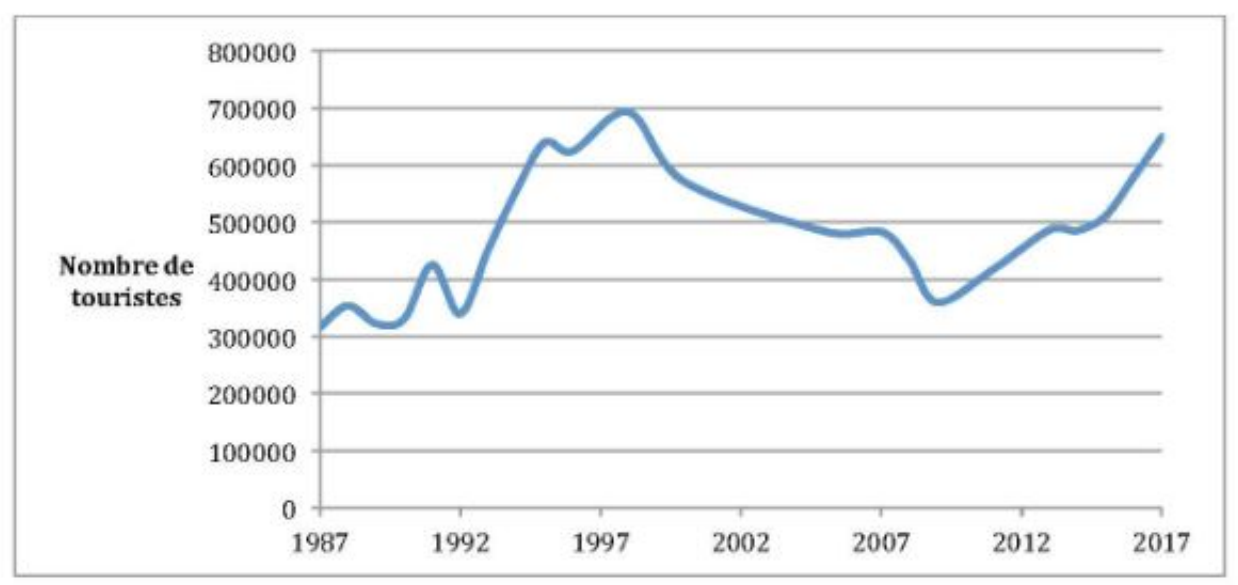

La « crise guadeloupéenne » révèle finalement les fragilités de la destination : difficulté à se démarquer de la concurrence des autres destinations caribéennes, remise en cause du «tout balnéaire ", forte dépendance de la clientèle française métropolitaine qui représente quasiment $90 \%$ de la fréquentation touristique totale, dégradation de l'image touristique... Mais elle conforte également le virage souhaité par certains acteurs du tourisme guadeloupéen en faveur des pratiques de tourisme et de sports de nature. Si les enquêtes annuelles de l'observatoire régional du Tourisme notent que pour une majorité des touristes, le soleil et les plages constituent encore le principal facteur attractif, la découverte de la nature apparaît régulièrement comme un atout complémentaire $^{3}$ (Observatoire régional du tourisme, 2018).

Dans un tel contexte, le Grand Cul-de-Sac Marin offre un cadre privilégié pour la diversification des pratiques de baignade mais surtout pour le développement des sports de nature. Les premières initiatives touristiques locales émergent à partir des années 1980 et, en 2000, les visiteurs sont presque 20000 à visiter annuellement la Réserve naturelle. 64 prestataires touristiques recensés (dont l'activité commerciale est déclarée) développent aujourd'hui des visites organisées dans l'Aire marine adjacente du Parc et en vivent en partie. Presque la moitié d'entre eux (44\%) ont leurs ports d'attache dans les communes riveraines de la baie majoritairement Sainte-Rose, BaieMahault et dans une moindre mesure Morne-à-l'eau, Petit-Canal, Port-Louis, Les Abymes et Le Lamentin. En revanche, ils sont $34,5 \%$ à déclarer un port d'attache sur la Riviera du Levant et $22 \%$ dans une commune de la Basse-Terre (fig. 5). La baie fournit donc un espace de pratiques touristiques, non pas seulement pour des prestataires qui y sont localisés et s'y sont développés souvent en complément d'activités préexistantes (pêche, restaurant, petite hôtellerie), mais aussi pour tout un ensemble d'acteurs économiques du tourisme qui interviennent traditionnellement sur l'espace maritime guadeloupéen.

Fig. 5 : Ports d'attache des prestataires touristiques opérant dans l'Aire marine adjacente du Parc national de Guadeloupe.

Source : N. Rollo, V. Andreu-Boussut, S. Charrier, C. Chadenas, C. Rufin-Soler 


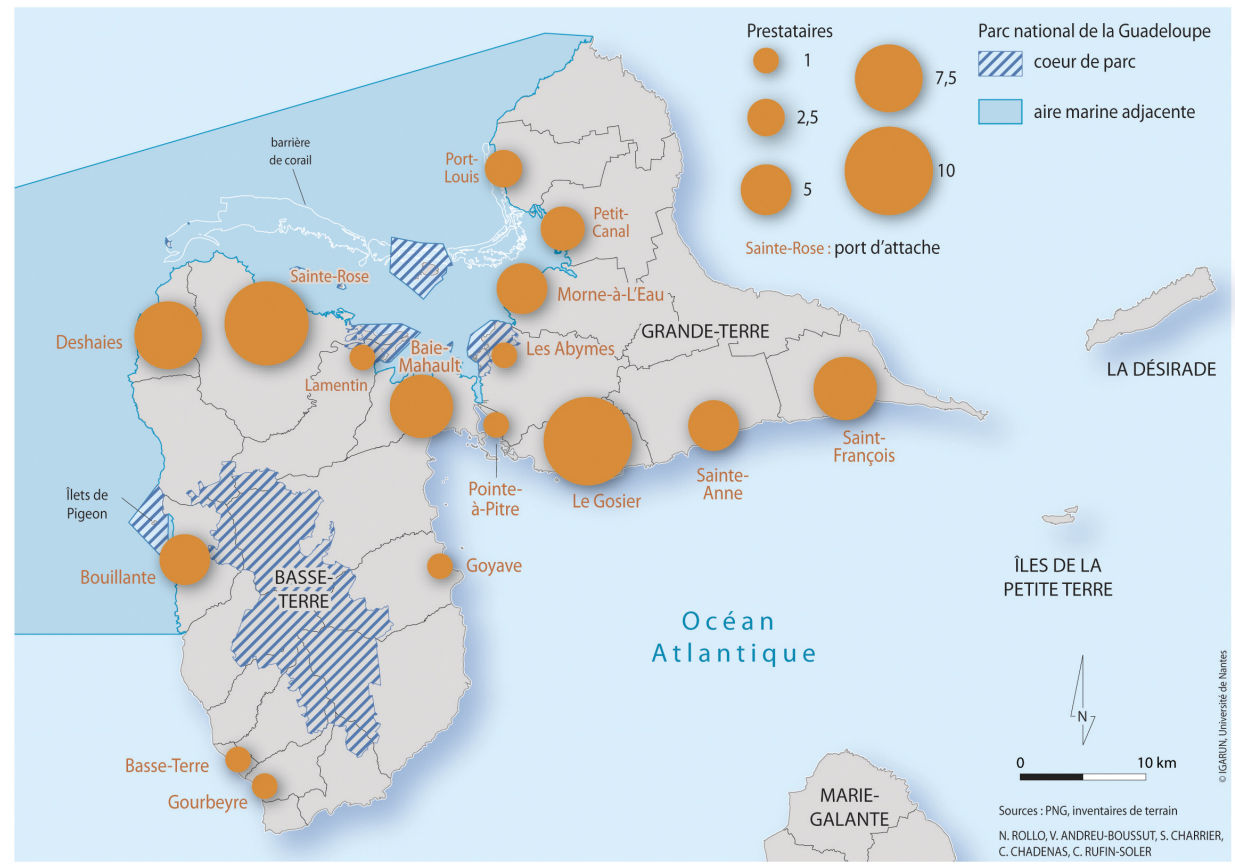

17 Parmi les prestations proposées, l'excursion reste de loin la pratique touristique dominante (fig. 6) car la plus adaptée au public familial qui constitue $80 \%$ des touristes en Guadeloupe (INSEE, 2014). Elle ferait d'ailleurs l'objet de plus de 10000 sorties d'embarcations en mer par an (Vaslet et al., 2013). Le «modèle » de l'excursion est à peu près le même pour l'ensemble des prestataires même s'il peut exister quelques variantes. Il se compose d'un itinéraire en bateau dont la destination finale est généralement un îlet où se baigner et où déjeuner; il peut comporter des pauses pour la baignade ou le PMT («Palmes, Masque, Tuba» ou snorkeling) et des visites des milieux naturels (découverte de la mangrove...). Si cinq prestataires proposent de la location de bateau avec ou sans skipper, il est clair que l'offre reste dans l'ensemble une offre de visites encadrées. Témoignant d'une trajectoire de diversification des pratiques, certains prestataires proposent désormais (fig. 6) la pêche (dont la pêche sous-marine), le jet ski (majoritairement pour les clientèles des hôtels de Saint-François et de Sainte-Anne), et plus récemment des activités sportives plus douces comme le kayak, la randonnée palmée, la plongée, le VTT des mers, le paddle, l'apnée... Une grande partie des excursions font ainsi de plus en plus la part belle à la découverte de la nature, en fonction des connaissances du personnel à bord, et certains prestataires en font une activité en tant que telle : stage de biologie marine, croisière d'observation des cétacés...

Fig. 6 : Types d'activités proposées par les prestataires touristiques dans l'Aire marine adjacente du Parc national de Guadeloupe.

V. Andreu-Boussut, C. Chadenas, C. Rufin-Soler 


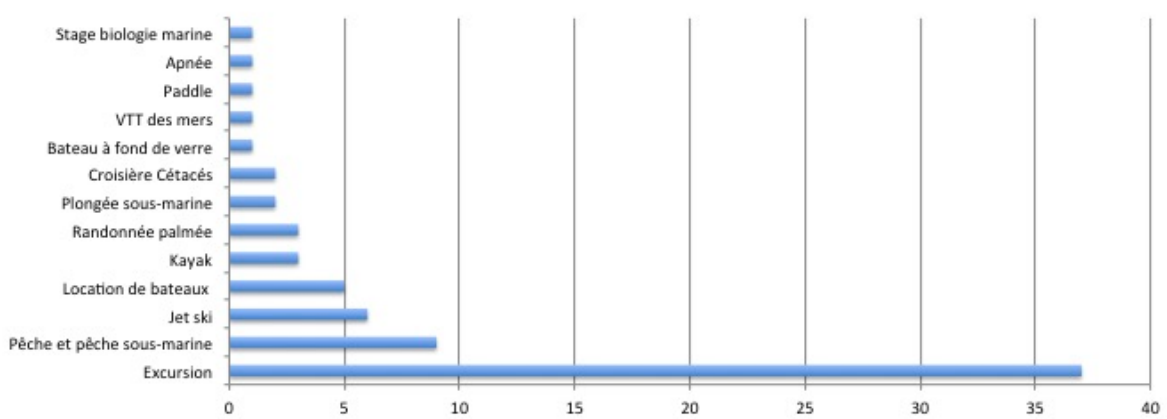

Beaucoup de prestataires utilisent des embarcations à moteur ${ }^{4}$, petites à moyennes, capables de transporter une dizaine de passagers. Au départ de Saint-François et Sainte-Anne, les professionnels utilisent plus spécifiquement des bateaux « cigarettes » puissamment motorisés pour le transport de groupes plus nombreux (fig. 7). A l'opposé, une dizaine de prestataires proposent des sorties à la voile, en bateau électrique et en zodiac.

Fig. 7 : Types d'embarcations proposées lors des excursions organisées par les prestataires touristiques dans l'Aire marine adjacente du Parc national de Guadeloupe.

V. Andreu-Boussut, C. Chadenas, C. Rufin-Soler 


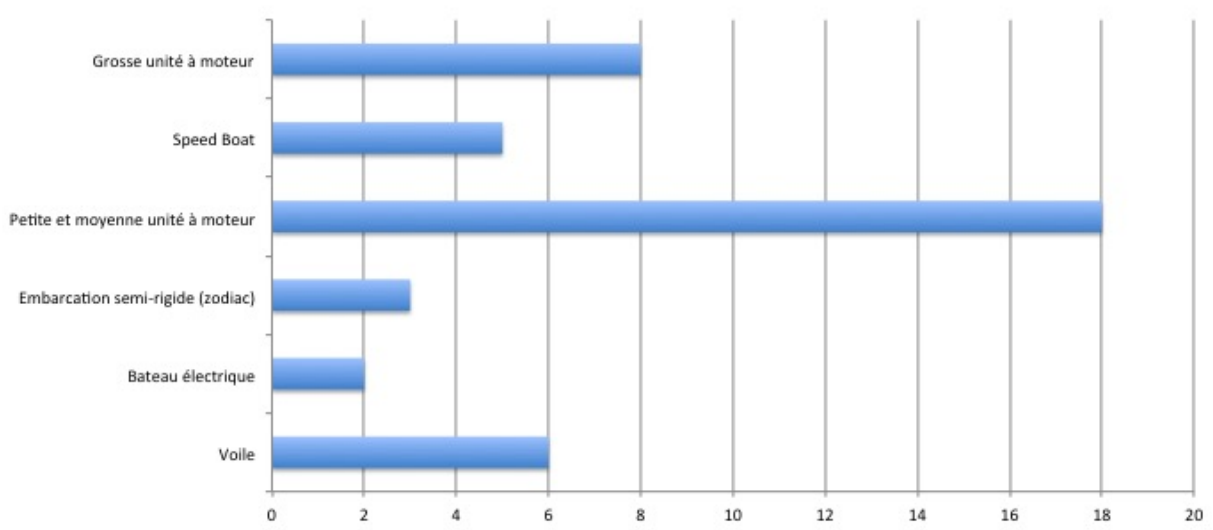
protection de la nature notamment parce que la fréquentation de l'Aire marine adjacente n'est pas soumise à autorisation. A l'inverse, 26 des 64 prestataires $(40,6 \%)$ sont autorisés par le Parc national à pénétrer dans les Cœurs dans le cadre des visites organisées qu'ils proposent. L'idée est ainsi de poursuivre le développement du tourisme de nature dans les espaces à plus forte valeur patrimoniale tout en tentant de maitriser les impacts des usages touristiques par le respect de la réglementation en vigueur (interdictions de pêcher, chasser, prélever faune ou flore, faire du bruit, faire $\mathrm{du}$ jet ski, faire du feu, camper...). Pour soutenir le développement des activités écotouristiques, le Parc national a néanmoins développé depuis 2004 la marque «Esprit Parc national ", déployée par les Parcs nationaux français en faveur du tourisme durable. Depuis l'extension du Parc en mer, sept prestataires ont fait le choix d'y adhérer. Si ce chiffre reste faible, certains prestataires indiquent que leur refus ne correspond pas à un manque d'intérêt en faveur du projet patrimonial, mais s'explique par une réticence à entrer dans un processus d'évaluation administrative lourde et par un manque de plus-value en matière de clientèle.

Une forte dualité structure caricaturalement les usages touristiques du Grand Cul-deSac Marin. D'un côté, une petite partie des prestataires, surtout installés sur la Riviera du Levant, fait le choix d'y développer une série de pratiques touristiques «traditionnelles » misant sur les sports «à sensation », de l'autre côté une autre petite partie de prestataires, installés dans les communes riveraines, tente de développer des pratiques "spécialisées" (plongée sous-marine, kayak, excursion à la voile) et respectueuses de la protection de la nature. Entre ces deux catégories différentes, la plus importante part des prestataires fait le choix d'excursions classiques intégrant à la fois pratiques balnéaires, pratiques traditionnelles (les grillades notamment) et interprétation des milieux naturels. Parmi ces derniers, des pêcheurs de la baie se sont reconvertis dans l'accompagnement des visiteurs. 


\section{2- Les enjeux de la gestion du tourisme dans un espace protégé : une conciliation nécessaire}

21 Si la patrimonialisation récente du Grand Cul-de-Sac Marin s'est accompagnée d'une intégration des usages traditionnels et touristiques, cette situation n'est pas sans poser un certain nombre de difficultés au Parc national dans la mise en œuvre du projet de protection.

\section{Des usages touristiques très concentrés dans l'espace...}

Si l'ensemble des pratiques spatiales déclarées par les visiteurs et les prestataires touristiques témoigne d'un usage dense du lagon, ces dernières se concentrent sur quelques points clés.

Pour les habitants fréquentant le lagon, quatre ports d'attache sont privilégiés : Rivière salée, Sainte-Rose, avant Morne Rouge et Baie Mahault. C'est surtout l'espace situé entre l'îlet La Biche et la " piscine ", l'̂llet Caret, et le sud/sud-ouest de l'îlet Fajou qui est le plus densément utilisé (fig. 8). Les habitants déclarent y venir fréquemment le week-end pour des activités « traditionnelles » de loisirs : baignade, PMT, pêche, piquenique et farniente. Le secteur ouest de Fajou est ainsi cité à de nombreuses reprises pour y déjeuner et y passer la nuit, à l'abri de l'îlet. Par ailleurs, la densité de parcours entre la Rivière salée et le reste de la baie correspond aux visiteurs qui partent des ports situés sur la Riviera du Levant, et profitent de la soirée ou du week-end pour aller dans des secteurs qu'ils ont l'habitude de fréquenter. Le plan d'eau situé entre les îlets Carénage et le port de Sainte-Rose, tout comme le nord de l'îlet à Christophe, représentent des espaces secondaires de destination, pour des parcours de visite moins longs et de proximité.

Fig. 8 : Synthèse des parcours de visite des habitants dans le Grand Cul-de-Sac Marin 


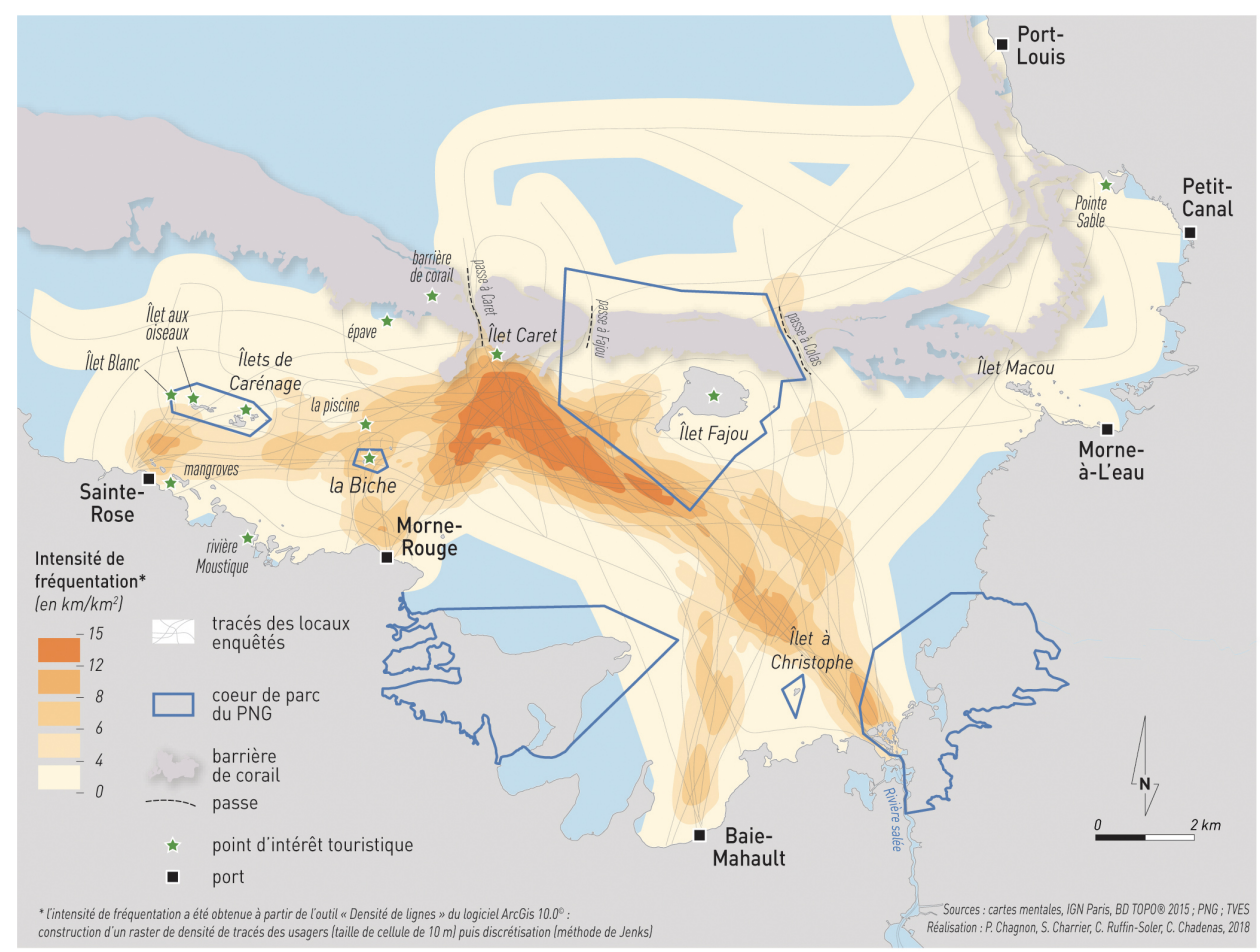

Pour les touristes ${ }^{5}$ visitant la baie, les parcours témoignent d'un effet de concentration relativement différent (fig. 9). Ainsi, trois espaces majeurs de destination sont identifiables : l'îlet La Biche et la « piscine » qui permettent la découverte des paysages sous-marins en PMT, l'îlet Caret, îlot de sable corallien situé à fleur d'eau pour la nage et le déjeuner, et le plan d'eau situé entre le port de Sainte-Rose et l'îlet Blanc. Plus secondaire, un autre espace de destination émerge néanmoins entre Morne-à-l'Eau et Petit Canal pour la découverte de la mangrove et éventuellement un pique-nique et une baignade à Pointe Sable. Ces pratiques touristiques renseignent sur les parcours privilégiés des prestataires, notamment ceux du port de Sainte-Rose, même si quelques touristes empruntent la Rivière salée depuis la Riviera du Levant. Rares sont les touristes qui viennent seuls dans le Grand Cul-de-Sac Marin, ils privilégient souvent un parcours de découverte organisé par un prestataire.

Fig. 9 : Synthèse des parcours de visite des touristes dans le Grand Cul-de-Sac Marin. 


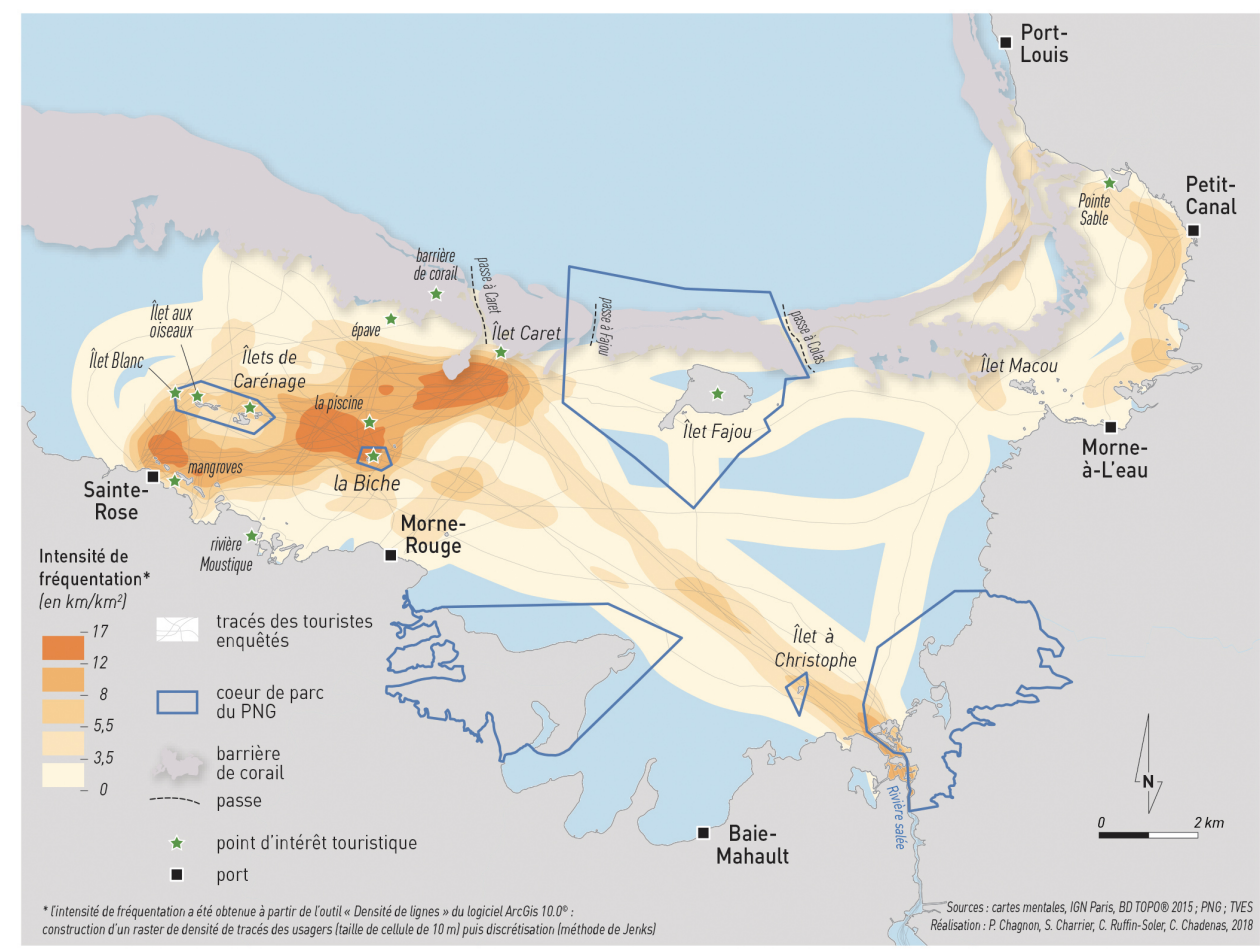

Puisque la majorité des touristes enquêtés l'ont été directement sur les îlets Caret, La Biche ou à Pointe Sable, il reste fondamental d'analyser également les parcours des prestataires touristiques rencontrés, qui rendent compte d'une variété plus grande des parcours de visite proposés et donc d'une plus grande dispersion à l'échelle de la baie. Pour ces derniers, le tracé de leurs visites montre qu'à partir de l'îlet Christophe, deux secteurs privilégiés de pratiques se distinguent (fig. 10). Un premier secteur, classique, concerne les îlets La Biche, Caret et leurs alentours (la "piscine ", "l'épave »). Le second, moins pratiqué, s'étire de l'îlet Macou à Pointe Sable en longeant la pente interne du récif. La moindre intensité de fréquentation de cette partie orientale du lagon témoigne néanmoins de l'intérêt grandissant des prestataires pour Pointe Sable et pour la découverte de la mangrove entre Petit-Canal et Morne-à-l'Eau. Support de pratiques nouvelles comme le kayak ou le VTT des mers, ce second espace de destination prend aussi de l'importance parce qu'il constitue une alternative à l'îlet Caret.

Fig. 10 : Synthèse des parcours de visite des prestataires touristiques dans le Grand Cul-de-Sac Marin.

Source : P. Chagnon, S. Charrier, C. Chadenas, C. Rufin-Soler, V. Andreu-Boussut 


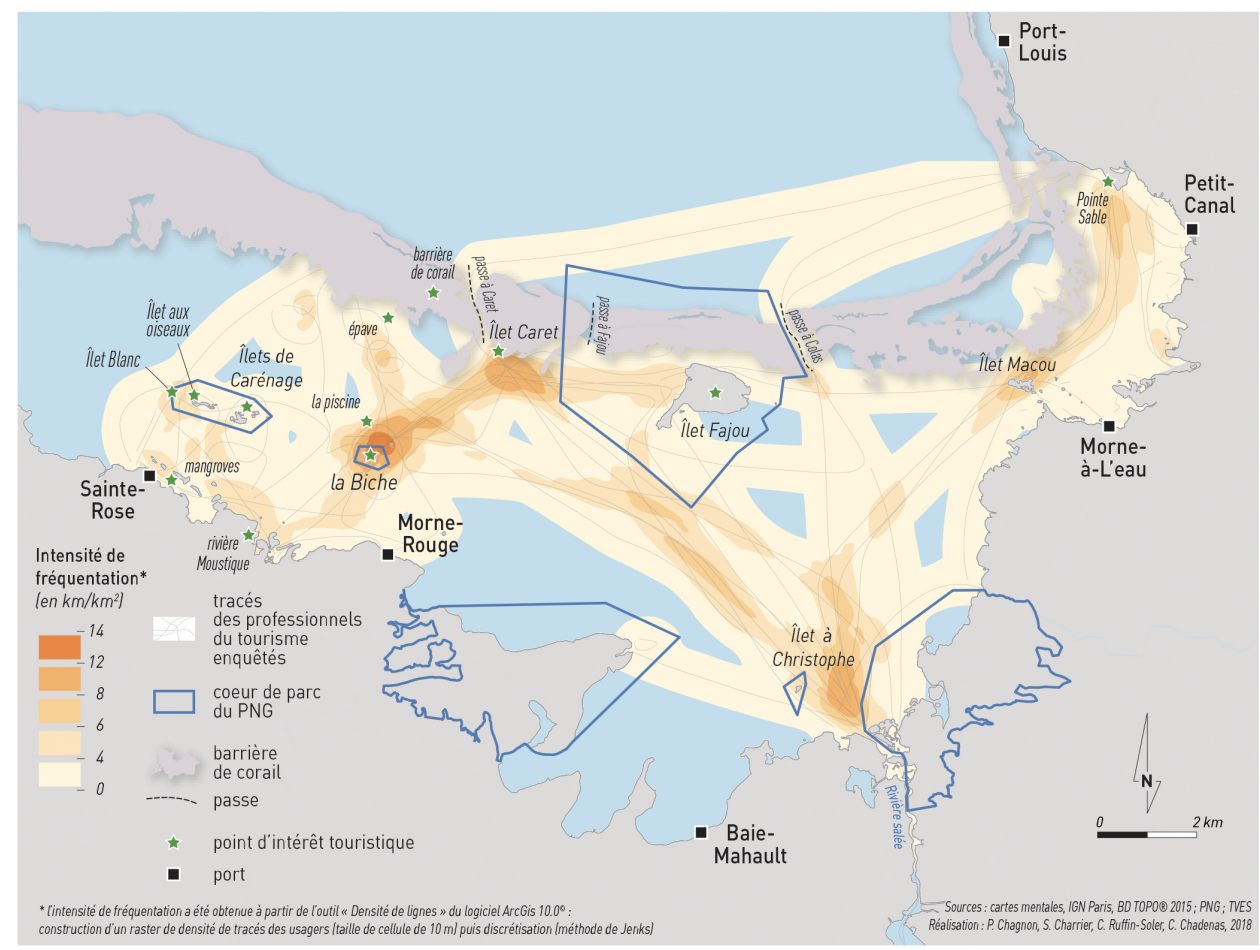

Les pratiques de visite de l'ensemble des usagers mettent clairement en évidence des secteurs de très forte fréquentation, véritables spots touristiques, près des Cœurs de Parc ou même à l'intérieur de leurs périmètres. En fait, quel que soit leur origine, les usagers semblent rechercher les mêmes paysages insulaires sous-marins des îles tropicales, même si certains habitants peuvent s'en éloigner pour chercher davantage de tranquillité, et sortent finalement très peu en pleine mer au-delà du récif.

\section{La nécessité de trouver de nouveaux modes de gestion plus durable}

Cette concentration de la fréquentation pose au moins trois séries de problèmes. La première concerne des conflits d'usage liés à l'exiguïté actuelle des îlets et des plages alors même que la baie est très vaste. Ces conflits peuvent se développer entre usagers aux pratiques difficilement compatibles mais qui partagent le même espace (conflits entre baigneurs et kite-surfers autour de l'îlet Caret) et entre habitués et prestataires touristiques (notamment pour les déjeuners dans les carbets). Dans ce dernier cas, s'il existe des pratiques agressives qui consistent à déposer un personnel le matin très tôt pour " privatiser » le carbet ou le morceau de plage jusqu'au moment de l'arrivée du groupe, les prestataires déclarent généralement s'entendre pour se répartir entre eux l'espace disponible ou selon un système de rotation temporelle (comme à l'îlet La Biche). Néanmoins, certains prestataires bien organisés et historiquement bien ancrés dans la baie disposent de concessions du domaine public, où ils peuvent aménager des carbets, comme à Pointe Sable en accord avec l'office national des Forêts.

La seconde série de problèmes concerne la très forte dépendance des pratiques touristiques à des îlets dont certains sont en train de démaigrir (îlet Caret, îlet Blanc) quand ils n'ont pas totalement disparu (îlet La Biche : fig. 11). Situés à fleur d'eau et directement soumis aux aléas météo-océaniques, ces îlets meubles, composés de sables, de galets ou de beach rock, parfois coiffés de palétuviers sont très mobiles et dépendent 
largement de facteurs extérieurs comme la puissance des vagues, la production sédimentaire au niveau du récif ou même sa morphologie... A ces facteurs naturels, s'ajoutent des facteurs anthropiques qui influent directement sur leur fonctionnement : les prélèvements sédimentaires effectués dans le lagon par le passé, les apports terrigènes, pollués par la culture de la canne, la décharge de la Gabarre et les communes riveraines, la pêche, l'urbanisation et la fréquentation touristique. D'une manière plus générale, les récifs coralliens sont en mauvais état écologique, souffrant d'une diminution de 30 à $50 \%$ de leur recouvrement (IFRECOR, 2016), si bien qu'ils ne jouent plus leurs rôles d'atténuateurs de la houle qui frappe ainsi plus durement les îlets situés dans le lagon. La baisse du taux de recouvrement corallien impacte également les espèces qui vivent en symbiose avec les coraux, et diminue leur apport sédimentaire. Evidemment, ces changements environnementaux systémiques interagissent avec certains effets du changement climatique attendus d'ici 2100 : élévation de la température moyenne en Guadeloupe de $1,6^{\circ} \mathrm{C}$ à $4,3^{\circ} \mathrm{C}$, élévation d'un mètre du niveau moyen de la mer, augmentation des précipitations en juillet... La principale conséquence qui en découle est une amplification des effets des submersions marines, surtout dans un contexte de fragilisation des mangroves qui jouent le rôle de zone tampon entre la terre et la mer.

Fig. 11 : Un carbet les pieds dans l'eau depuis la disparition de l'îlet La Biche

Photo juin 2015, Céline Chadenas

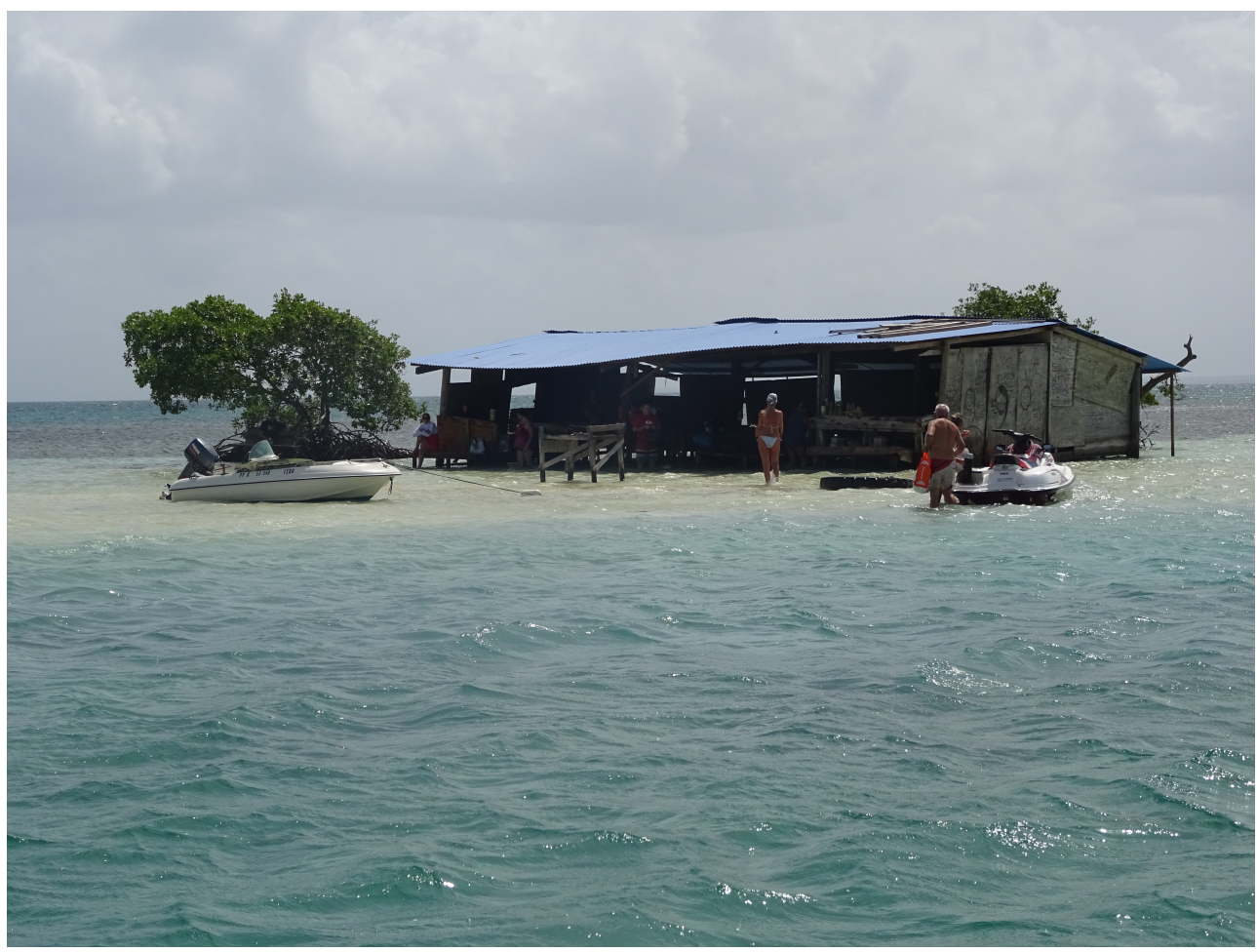

Enfin, la troisième série de problèmes concerne plus spécifiquement les impacts environnementaux liés aux pratiques des visiteurs: plongeurs et baigneurs qui marchent ou touchent accidentellement les coraux, plaisanciers dont les ancres déciment les herbiers de phanérogames marines, visiteurs des îlets qui piétinent la végétation ou empêchent la nidification des oiseaux (comme sur les plages des îlets Carénage où nichent petites Sternes et Sternes Pierregarin)... Symbole du tourisme en 
Guadeloupe, l'îlet Caret incarne parfaitement à lui-seul ces trois types d'enjeux imbriqués. Très mobile sur son platier, il a été fixé par des aménagements en dur (digue, épis) pour enrayer une érosion toujours plus rapide. Si certains usagers considèrent les prélèvements sédimentaires ou le changement climatique responsables de son état actuel, de récentes études scientifiques considèrent inversement que seul l'homme est responsable de sa situation (BRGM, 2016). L'érosion serait avant tout liée à une pression anthropique trop importante qui empêcherait d'une part, la végétation de fixer le sable nouvellement accumulé et, d'autre part, les herbiers de phanérogames, régulièrement arrachés par les ancres, de fixer les sédiments... (BRGM, 2016). Ainsi, l'îlet Caret est au centre de l'attention, victime de son succès. Réduit aujourd'hui à une accumulation de sable fin, l'évolution rapide de sa morphologie questionne sérieusement son avenir touristique. Les habitués de la baie, qui y sont très attachés, sont aujourd'hui inquiets quant à son devenir, à l'image de la pétition pour "la sauvegarde de l'îlet Caret » lancée en 2016 sur internet et qui a reçu 3821 signatures.

Si la pression sociale est forte pour défendre les îlets contre l'érosion, les solutions à trouver pour une meilleure gestion des usages touristiques restent largement discutées aujourd'hui. Trois voies ont été choisies : i) les pratiques les plus impactantes pour l'environnement font progressivement l'objet d'une régulation ou d'une interdiction (comme les Mercury Days 6 ), ii) la fréquentation de certains espaces en Cœurs de Parc peut être soumise à limitation comme à l'îlet Blanc où un arrêté préfectoral interdit le débarquement et la navigation à moins de 100 mètres du $1^{\mathrm{er}}$ mai au 31 août chaque année pour permettre la reproduction des sternes, iii) une charte de bonne conduite des plaisanciers est en préparation avec le Guadeloupe Marlin Club.

\section{Se réconcilier avec les habitants et les usagers : un Parc régulateur mais pas gendarme}

31 Le positionnement actuel du Parc national est désormais sur la corde raide. D'un côté, il doit rompre avec une tradition qui laissait initialement peu de place à la négociation avec les habitants et les collectivités locales et mettre en œuvre une plus grande concertation (loi de 2006 et nouvelle charte de territoire de 2009). Et d'un autre côté, il conserve évidemment ses missions fondamentales de conservation de la biodiversité dans les Cœurs de Parc, notamment pour le rôle de nourricerie que ces derniers jouent dans le maintien des stocks de pêche.

Jusque dans les années 2000, le Parc national est régulièrement critiqué pour la grande défiance qu'il inspire aux habitants. Subissant de plein fouet les restrictions d'usages traditionnels pour des raisons de conservation écologique et bénéficiant rarement des retombées économiques des nouvelles activités touristiques, ces communautés locales pâtissent d'une justice spatiale clairement en leur défaveur (Lapointe et Gagnon, 2009, 2012). En effet, le Parc est initialement bâti sur un massif forestier, historiquement propriété du département gérée par l'office national des Forêts, qui n'a jamais nécessité l'intégration des collectivités locales dans le projet de protection et de gestion. Le nouveau Parc national de 2009 marque un tournant majeur puisqu'il donne non seulement un rôle plus important aux communes dans sa gouvernance et son fonctionnement, mais enclenche également une concertation plus grande lors de l'élaboration de ses projets de gestion et d'aménagement. La nomination à cette époque 
d'un Guadeloupéen comme nouveau directeur est un signal fort qui vient renforcer la légitimité du Parc national.

Néanmoins, à l'échelle du Grand Cul-de-Sac Marin, le respect de l'interdiction de la pêche dans les Cœurs de Parc, tout comme celui des périodes de pêche et des tailles des captures restent une question épineuse. Ainsi, même si pour le Parc la sanction n'est pas une fin en soi, elle est nécessaire pour faire respecter la protection de la biodiversité mais aussi pour maintenir une forme d'équité de traitement entre pêcheurs. La stratégie est alors de combiner un effort de communication et de sensibilisation en direction des usagers tout en maintenant une surveillance accrue, en collaboration avec les communes. Face au constat du manque de personnel en capacité de veiller en permanence sur le respect de la réglementation, le Parc national déplore régulièrement le manque de temps disponible pour mener à bien un travail fin de sensibilisation systématique des usagers à la protection du lagon.

Cette concertation engagée auprès des communes à la fois sur les territoires de l'Aire d'adhésion et de l'Aire marine adjacente fait l'objet d'un retour majoritairement positif de la part des acteurs institutionnels, d'une grande partie des communes et des acteurs associatifs. Le sentiment général de l'évolution des habitudes de travail est d'autant plus fort lorsque des partenariats sont formalisés. Certaines des collectivités locales estiment d'ailleurs que cette concertation est indispensable à la bonne gestion des usages : « le but n'est pas d'entrer en conflit avec les usagers [...]. Si on arrive de but en blanc et on impose les choses là on va droit au clash " (entretien avec la Mairie de Sainte-Rose, le 13 mars 2018). Et s'il n'est pas simple d'aller directement au-devant des usagers, les liens avec les associations qui les représentent semblent s'améliorer grâce aux réunions mises en place.

Le lien qu'entretiennent les prestataires touristiques avec le Parc national est à l'inverse plus complexe. Si depuis 2009 les autorisations d'accès aux Cœurs de Parc ont fait l'objet d'un effort important de contrôle et de régulation, ces démarches ont aussi rapproché un certain nombre des professionnels de l'équipe du Parc permettant d'aller vers une meilleure interconnaissance. Contrairement aux relations établies avec les collectivités locales, il n'existe pas aujourd'hui ni de lieu ni d'instance de concertation avec les prestataires touristiques. Des ateliers et des réunions de travail sont régulièrement proposés mais se heurtent au grand nombre et à l'éclatement de ces professionnels, qui ne disposent pas d'une représentation syndicale commune. Ainsi, certains d'entre eux notent une évolution positive facilitée par des réunions collectives inédites et soulignent la plus-value du Parc dans son accompagnement en matière d'interprétation des milieux écologiques. Si l'effort de dialogue insufflé par le Parc est jugé trop lent pour la plupart des professionnels, ils estiment néanmoins qu'ils sont désormais davantage entendus : « en général c'est eux qui décident mais maintenant on leur met un peu la pression [...], c'est en train de se faire, ils ont fait des ateliers, ils écoutent nos doléances" (entretien avec un prestataire touristique de Saint-François, le 19 février 2018). Cela étant, une majorité d'entre eux continue de manifester une grande défiance considérant la concertation comme un processus de façade pour faire accepter des décisions préconçues en amont : « on a souvent des réunions qui tournent un petit peu en rond [...], on nous convoque [...] mais les décisions sont déjà prises donc c'est juste pour nous faire croire qu'on intervient » (entretien avec un prestataire de kayak, le $1^{\text {er }}$ mars 2018), «il y a des réunions de travail mais la plupart du temps on a l'impression que les dés sont déjà pipés, on sent que c'est orienté » (entretien avec un 
prestataire de Sainte-Anne, le 16 février 2018). Néanmoins, la plupart des professionnels sont demandeurs d'une meilleure concertation et regrettent régulièrement de ne pas être considérés comme de vrais partenaires : «je ne crois pas qu'ils travaillent assez avec les professionnels, je pense que le Parc plutôt que de nous fliquer, devrait nous considérer comme des partenaires » (entretien avec un prestataire de Morne-Rouge, le 15 janvier 2018). Certains des prestataires touristiques déclarent ainsi leur difficulté à participer davantage au projet de protection à cause de l'image que le Parc peut avoir d'eux.

Dans les discours des acteurs locaux émerge finalement le besoin d'une instance de gestion collaborative à l'échelle de la baie qui pourrait regrouper toutes les parties prenantes. Cet outil de gouvernance serait alors, non pas seulement un espace de sensibilisation ou d'échange libre, mais un espace de construction d'un diagnostic partagé de la situation écologique du Grand Cul-de-Sac Marin et des solutions à mettre collectivement en œuvre.

\section{3- Trois postures différenciées sur le degré de protection et l'ouverture au tourisme : des visions réconciliables?}

Sur la recherche d'un équilibre à trouver entre protection et tourisme, trois postures coexistent aujourd'hui dans le lagon, qui transcendent largement les catégories d'acteurs territoriaux. Ces visions s'enracinent dans des représentations de la nature qui opposent nature et culture en privilégiant la défense de l'un ou de l'autre (anthropocentrisme, biocentrisme), ou qui intègrent les deux dans une même communauté du vivant (écocentrisme) (Depraz, 2008).

\section{Une posture anthropocentrée qui voit le Grand Cul-de-Sac Marin comme un espace économique}

Une partie des prestataires et des habitants, plus rarement quelques collectivités locales, considèrent le Grand Cul-de-Sac Marin davantage comme un espace économique, un ensemble de ressources, sans remettre totalement en cause la protection qui y est instaurée. Cette posture s'articule à un argumentaire qui n'attribue pas la dégradation constatée des écosystèmes à la fréquentation touristique, même parmi les usagers les plus habitués. Evidemment le discours des prestataires touristiques sur cette thématique doit être pris avec précaution puisqu'ils ont peu d'intérêt à ce que le tourisme soit rendu seul responsable de la dégradation environnementale. Pour autant, le démaigrissement des îlets est régulièrement jugé comme un phénomène grave, non pas pour des raisons écologiques, mais pour des raisons économiques.

Dans la continuité de ce discours, la fréquentation touristique n'est pas jugée excessive sauf parfois sur l'îlet Caret où de façon ponctuelle : « la semaine c'est plutôt calme, il y a trop de monde le week-end [...], c'est très bien, les gens ils viennent. Il y a beaucoup de touristes je trouve que c'est bien » (entretien avec un touriste, rencontré le 19 juillet 2017), «il y avait beaucoup plus de monde avant [...], mais sur l'îlet même il y a beaucoup moins de fréquentation, entre 10 heures et midi, il y a du monde, il 
recommence à y avoir du monde de $14 \mathrm{~h}$ à $15 \mathrm{~h} 30$ environ, mais il y a beaucoup moins de fréquentation qu'il y en a eu - il y a plus de bateaux et il y a moins de gens sur les îlets " (entretien avec le cluster maritime de Guadeloupe, le 7 juin 2017). Ainsi cette fréquentation est considérée très positivement pour ses effets économiques : «c'est un atout touristique pour attirer du monde sur Port-Louis [...], oui dans la mesure où sur le plan économique ça peut permettre le développement économique pour le Nord Grande-Terre c'est un plus pour nous » (entretien avec la mairie de Port-Louis, le 10 juillet 2017). Dès lors, certains prestataires et collectivités ambitionnent d'aller plus loin dans la valorisation touristique et les aménagements: "nous sommes en passe d'avoir une compétence sur les îlets en tant que gestionnaire des milieux naturels [...] qui permettraient de valoriser et d'aménager les milieux, de créer des activités économiques [...]. Nous voulons être acteurs de notre développement sur ces espaces naturels " (entretien avec la mairie de Petit-Canal, le 8 juin 2017). Cette posture aménagiste peut parfois remettre en cause le projet de protection, niant la nécessité d'intervenir pour enrayer la dégradation environnementale: «il faut déboiser, valoriser pour l'usage économique, laisser les choses naturellement comme c'est là, aménager pour que les gens puissent venir, y'a pas de réglementation à faire " (entretien avec un prestataire touristique de Port-Louis, le 28 février 2018).

Majoritairement, la conservation des îlets reste une préoccupation et nombre de solutions sont envisagées pour enrayer l'évolution régressive de ces paysages (disparition des îlets, dégradation du récif). Pour l'îlet Caret, le rechargement sédimentaire, réalisé sur d'autres sites guadeloupéens, est régulièrement évoqué comme une solution «naturelle» et douce. Quant à la restauration du récif, elle pourrait passer par le bouturage de coraux et par une meilleure gestion de la qualité de l'eau, incriminant au passage les activités agricoles.

\section{Une posture écocentrée favorable à un renforcement de la protection et à une régulation des usages touristiques}

41 La posture la plus fréquente, portée par quelques prestataires et une majorité d'habitants, de touristes et de collectivités locales, consiste à souhaiter un meilleur équilibre entre usages touristiques et protection. Cette vision s'enracine dans un argumentaire qui considère la fréquentation touristique comme responsable d'un certain nombre d'impacts environnementaux, qualifiée à l'occasion de surfréquentation. La capacité de charge environnementale et sociale des lieux les plus visités serait alors dépassée : « on est dans une saison qui est plutôt creuse et je trouve qu'on est déjà beaucoup [...] C'est de la sur-fréquentation oui [...] un îlet comme Caret aujourd'hui je pense qu'il est plus en mesure de supporter ce qu'il se passe » (entretien avec un touriste, le 28 juillet 2017), « ça m'a surpris à Caret de voir les mecs avec tente et barbecue, ça m'a un peu interpellé par rapport à l'environnement du Parc» (entretien avec un touriste, 26 décembre 2017). Si le démaigrissement des îlets n'est pas toujours imputé aux touristes, il n'en demeure pas moins que les traces des visiteurs dans le milieu naturel sont jugées sévèrement : «je pense que c'est pas la fréquentation qui fait le problème, un îlet ça bouge, le problème de la fréquentation c'est la pollution, les déchets» (entretien avec un habitant, le 12 juin 2017). La fréquentation est d'ailleurs très majoritairement considérée à la hausse : « depuis 4 ans, je trouve qu'il y a 
vraiment de la part des professionnels une explosion, notamment des unités qui partent de Sainte-Rose » (entretien avec un habitant, 24 février 2018).

Malgré ce constat, l'idée dominante est que la recherche d'un compromis avec la protection est indispensable parce qu'il est légitime pour les visiteurs d'avoir accès à l'espace patrimonial et que des acteurs économiques en vivent: «le must pour l'environnement ce serait de tout interdire mais pour le tourisme c'est pas possible, donc il faut trouver un juste milieu, le bon compromis » (entretien avec un prestataire touristique de Sainte-Rose, le 5 juillet 2017). Dans cette perspective, les concessions proposées vont relativement loin. Certains n'hésitent pas à souhaiter une réglementation plus forte et contraignante accompagnée d'une démarche accrue de sensibilisation et d'éducation : « pour les îlets, il faudrait que ce soit très réglementé, de la communication pour éviter que les gens touchent les coquillages, les plantes [...] prévoir des personnes qui vivraient sur certains îlets peut-être en permanence ou qui seraient là par roulement, qu'il y ait une présence du Parc» (entretien avec l'association Les Amis de la Baie, le 5 juillet 2017). La limitation de la fréquentation est aussi clairement avancée comme solution possible. Ainsi, des quotas pourraient être établis sur le nombre de visiteurs ou sur le nombre de prestataires touristiques autorisés, ou des fermetures ponctuelles pourraient être mises en place à des moments particuliers de l'année afin de laisser un temps de repos aux écosystèmes : « ça pourrait être intéressant, comme à Petite-Terre, il y a des quotas. Comme au Pérou, le Macchu Pichu il y a des quotas. Toutes les belles choses à voir il y a des quotas en fait [...] ça permet de préserver les lieux, c'est pas choquant » (entretien avec un touriste, le 5 janvier 2018), "il faut limiter [...] voir combien un îlet peut recevoir par jour et réglementer fonction de ça et ne pas donner une accréditation à tout le monde " (entretien avec le Guadeloupe Marlin Club, le 25 mai 2017). Cette proposition de quotas, évoquée par les touristes, porte à débat pour certains professionnels qui craignent les conflits et revendiquent un droit d'accès pour tous au lagon: "ce qui me pose problème dans les quotas c'est qu'on va tomber dans un tourisme sélectif » (entretien avec un touriste, le 28 juillet 2017).

Le report des usages touristiques sur d'autres secteurs moins sensibles écologiquement, y compris à l'extérieur du Grand Cul-de-Sac Marin, est régulièrement évoqué par quelques prestataires et par de nombreux habitués: «personnellement limiter mon accès ou aller ailleurs essayer de faire autre chose [...] on a d'autres archipels, il y a la Désirade, les Saintes, Marie-Galante, bon c'est un peu plus loin, après il faut se limiter pour avoir une préservation, moi en tout cas je suis prêt à le faire » (entretien avec un touriste, le 5 août 2017). En l'occurrence certains usagers ont déjà reporté leurs pratiques ailleurs, notamment en allant à Pointe Sable (fig. 12).

Fig. 12 : Calme, cocotiers et sable blanc: Pointe Sable offre tout ce que les touristes recherchent et constitue une destination alternative dans le lagon

Photo février 2018, Céline Chadenas 


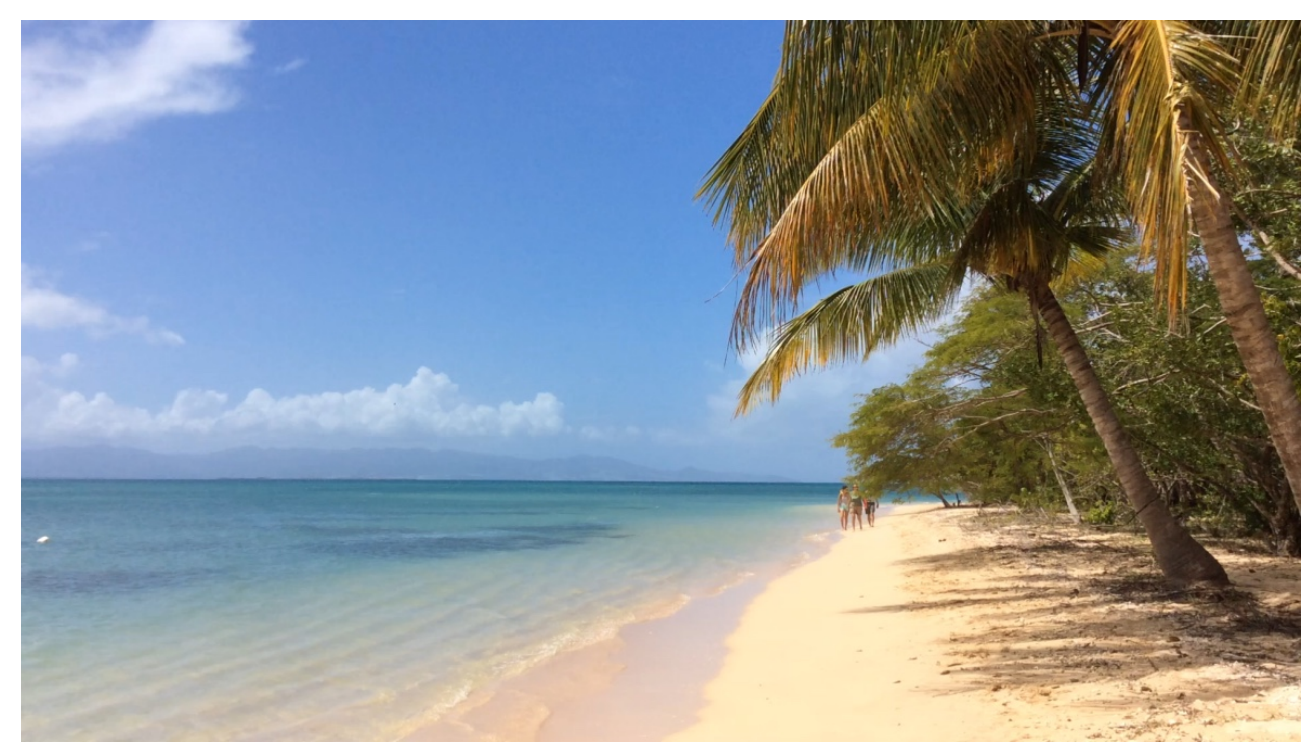

Préalable à une meilleure régulation des flux touristiques, des mesures en faveur d'une reconquête de l'état écologique du Grand Cul-de-Sac Marin sont largement plébiscitées. Au-delà des solutions à imaginer pour lutter contre l'érosion des îlets, des aménagements pour l'accueil du public sont plus particulièrement souhaités. Même si cette posture est paradoxale pour un espace protégé, les équipements évoqués visent à réduire les impacts écologiques des flux de visiteurs et à mieux sensibiliser le public sur les comportements à avoir dans le lagon: des panneaux d'information et d'interprétation, des sentiers pédagogiques, des sentiers PMT, des places à feu, et surtout des corps-morts, qui évitent aux bateaux d'utiliser leurs ancres et de dégrader ainsi les herbiers. Pour les acteurs de la protection (Parc national, office national des Forêts), le choix des aménagements peut être au service de la protection quand ces derniers permettent de calibrer les limites des flux souhaitables de visiteurs et leur extension spatiale.

\section{Une posture biocentrée favorable à un renforcement maximal de la protection}

Une dernière posture, favorable à un renforcement de la protection de la baie, émane d'une plus faible part des habitants et des touristes, mais aussi des prestataires touristiques, qui l'énoncent généralement avec une grande conviction. L'argumentaire qui soutient ce positionnement est triple. Il attribue d'abord une valeur très élevée aux milieux écologiques et témoigne ainsi d'un très fort attachement aux lieux. Il est très pessimiste sur le mauvais état écologique du lagon pour lequel la pêche et le tourisme sont jugés très sévèrement. Et il est très critique sur la protection considérée comme largement insuffisante. En l'occurrence, plusieurs d'entre eux montrent une réelle inquiétude sur la pression grandissante du tourisme sur le lagon, n'hésitant pas à déployer un discours très alarmiste. Certains pensent que la réglementation n'est pas assez contraignante: "pour moi si on protège il faudrait que ce soit interdit, pas donner des autorisations à certains [...] laisser un temps de repos, de reconstruction » (entretien avec un prestataire touristique de Sainte-Rose, 13 juillet 2017). La communication du Parc national est jugée insuffisante et l'inconscience et l'ignorance des usagers est fustigée. : "personne ne le sait que c'est un espace naturel protégé. 
Même un plaisancier. Il devrait savoir que quand tu vas dans le Grand Cul-de-sac Marin, tu ne jettes pas l'ancre, ou alors dans le sable [...]. Il faut le mettre en cœur de Parc (entretien avec un guide de pêche, le 28 février 2018). Même le Parc national revient régulièrement sur sa difficulté à faire respecter la loi dans les Cœurs. :

ce qui fonde quand même l'existence des Parcs nationaux, c'est les cœurs, c'est les territoires qui ont été considérés comme exceptionnels au regard de la biologie, des paysages, du caractère et ça pour moi c'est pas transigeable, [...] si donc on n'arrive même pas à atteindre cet objectif d'amélioration des peuplements ichtyologiques qui sont dans la réserve je ne vois pas à quoi on sert (entretien exploratoire avec le Parc national, mai 2015).

La préconisation consiste donc en une sanctuarisation complète de l'espace et une position préservationniste contre les usages de pêche et régulièrement contre les usages touristiques. Pour l'un des prestataires rencontrés, cet engagement peut être très fort, même si toute son activité professionnelle s'exerce dans le Grand Cul-de-Sac Marin. :

J'hallucine qu'on ne protège pas, qu'on ne dise pas c'est interdit de pêcher. Point barre. Le jour où on me le dit, je fais autre chose. Ce serait une bonne nouvelle [...]. Et quand tu veux y aller, et que tu es un professionnel de la mer, que tu sois obligé de cotiser au Parc avec un mec qui contrôle (entretien avec un guide de pêche, le 28 février 2018).

Compte tenu d'une telle vision, il n'y a pas besoin d'aménagements supplémentaires dans le lagon, l'objectif est de laisser le moins possible d'empreinte humaine sur les milieux naturels en libre évolution.

\section{Conclusion}

Les visions des acteurs locaux au sujet de l'espace protégé sont loin d'être irréconciliables même si elles diffèrent sur la place des usages touristiques. Elles témoignent d'une appropriation patrimoniale bien partagée et d'un grand attachement aux îlets ou au milieu naturel dans sa globalité. Le débat est donc celui du degré de protection et des moyens de gestion à choisir pour limiter les impacts environnementaux des usages touristiques.

Dix ans après la création de l'Aire marine adjacente, l'heure est désormais à la recherche d'une meilleure gestion des usages touristiques. Si le Parc national ne remet pas en cause l'ouverture au tourisme, il souhaite néanmoins infléchir l'image de la baie comme espace de loisirs vers celle d'un espace protégé. Le projet de réintroduction du Lamantin des Caraïbes participe de cet objectif. Ce mammifère marin jouerait le rôle d'ambassadeur du Parc national en mer et servirait d'indicateur de la qualité des milieux écologiques par sa fonction d'espèce parapluie. S'il n'est pas question actuellement de prélever un droit d'entrée ou de limiter l'accès des îlets, une réflexion sur une meilleure organisation des usages à l'échelle de la baie a été récemment confiée au bureau d'études Caraïbes Environnement. L'objectif est de maîtriser la fréquentation en redistribuant les flux de visiteurs à l'écart des Cœurs de Parc et en réfléchissant à un zonage des vocations et des usages. En parallèle, l'écotaxe sur les passagers maritimes dans les espaces protégés, dite Taxe Barnier de 1995, fait l'objet d'une négociation pour sa mise en place progressive en $2018^{7}$. Même si les professionnels comprennent l'intérêt de faire participer financièrement les visiteurs aux actions de protection, ils estiment in 
fine qu'ils en seront les financeurs et souhaiteraient davantage de pouvoir de décision dans les mesures de gestion.

Dans le Grand Cul-de-Sac Marin, si les professionnels sont plutôt favorables à l'idée de négocier un code de conduite commun et un zonage des usages (comme dans le Parc marin du Saguenay-Saint-Laurent au Québec ou le Parc national de la mer des Wadden au Danemark), les habitants comme les touristes sont néanmoins favorables au renforcement de la réglementation à l'intérieur du Parc national. Il reste donc à construire une gouvernance adéquate que tous les acteurs locaux appellent de leurs vœux.

\section{BIBLIOGRAPHIE}

Andreu-Boussut, Vincent, Salin, Elodie, et Chadenas, Céline, « Sustaining Natural Heritage Sites as Great Tourist Attractions : International Guidelines versus Local Contexts », in Lia Bassa (dir.), Proceedings of Tourism and Cultural Landscapes Conference, Budapest, 12-16 juin 2016, Volume 8, Budapest, 2016, p . 8-27.

Bouin, Frédéric, « Tourisme et environnement : atouts pour l'engagement d'une démarche de développement durable en Guadeloupe », in Jean-Marie Breton (dir.), Tourisme, environnement et aires protégées (Antilles, Guyane, Haïti, Québec), Ed. Karthala, 2004, pp. 329-340.

Breton, Jean-Marie (dir.), Tourisme, environnement et aires protégées, Ed. Karthala, 2004, 524 p.

BRGM, Diagnostic et propositions de gestion de l'érosion de l'îlet Caret, 2016, 17 p.

Buttifant, Audrey, Mège, Simone, Marie, Alain, Delloue, Xavier, et Vincent, Cédric,Plan de gestion Réserve naturelle du Grand Cul-de-sac Marin, Parc national de Guadeloupe et Université des Antilles et de la Guyane, 2008, $418 \mathrm{p}$.

Camus, Martine, et Neiss, Martine, Insee Analyses Guadeloupe, n² 2, INSEE, 2014, 4 p.

Dehoorne, Olivier, « Tourisme durable et écotourisme. Le positionnement des destinations caribéennes ", in Jean-Marie Breton (dir.), Tourisme durable et patrimoines. Une dialectique développementale ? (Europe, Caraibe, Amériques, Afrique, Asie), Ed. Karthala, 2011, p. 385-399.

Desvergnes, Manuel, et Vincent, Cédric, « Comment concilier valorisation touristique et protection de l'écosystème mangrove dans la baie du Grand Cul-de-Sac Marin (Guadeloupe) ? ", in Jean-Marie Breton (dir.), Tourisme durable et patrimoines. Une dialectique développementale? (Europe, Caraibe, Amériques, Afrique, Asie), Ed. Karthala, 2011, p. 401-421.

Eagles, Paul F. J., McCool, Stephen F., et Haynes, Christopher D. A., Sustainable Tourism in Protected Areas: Guidelines for Planning and Management, Best Practice Protected Area Guidelines Series $\mathrm{N}^{\circ} 8$, IUCN, Gland, 2002, 183 p.

Féral, François, «L'extension récente de la taille des aires marines protégées : une progression des surfaces inversement proportionnelle à leur normativité ", Vertigo - la revue électronique en sciences de l'environnement [En ligne], Hors-série 9 | Juillet 2011, mis en ligne le 13 juillet 2011, page consultée le 26 avril 2018. 
Foxlee, Jasmine, « Key principles and directions for tourism in protected areas: a review of existing charters, guidelines and declarations ", in Robyn Bushell (ed.), Tourism and Protected Areas: benefits beyond boundaries, Vth IUCN World Parks Congress, CABI, Wellington, 2007, p. 44-70. Grandbois, Michel, «Ecotourisme et aires protégées littorales et maritimes en Guadeloupe et en Martinique ", in Jean-Marie Breton (dir.), Tourisme, environnement et aires protégées (Antilles, Guyane, Haïti, Québec), Ed. Karthala, 2004, p. 111-128.

Grenier, Christophe, Conservation contre nature : les îles Galapagos, IRD Editions, 2000, 322 p.

IFRECOR, Etat des récifs coralliens et des écosystèmes associés des Outre-mer français en 2015, 2016, $168 \mathrm{p}$.

Lapointe, Dominic, et Gagnon, Christiane, « Conservation et écotourisme : une lecture par la justice environnementale du cas des communautés voisines du Parc national de la Guadeloupe », Études caribéennes [En ligne], 12 | Avril 2009, mis en ligne le 15 avril 2009, page consultée le 24 avril 2018.

Lapointe, Dominic, et Gagnon, Christiane, «A l'ombre des parcs : la conservation comme enjeu de justice environnementale pour les communautés locales? », in David Blanchon, Jean Gardin et Sophie Moreau (dir.), Justice et injustices environnementales, Presses universitaires de Paris Nanterre, 2012, p. 149-169.

Leung, Yu-Fai, Spenceley, Anna, Hvenegaard, Glen, and Buckley, Ralf, Tourism and visitor management in protected areas. Guidelines for sustainability, IUCN, Craig Groves editor, 2015, $240 \mathrm{p}$.

Meur-Férec, Catherine, « Entre surfréquentation et sanctuarisation des espaces littoraux de nature ", L'Espace géographique, Tome 36, 2007, p. 41-50.

Observatoire régional du Tourisme, Typologie des clientèles touristiques de la destination Guadeloupe, Région Guadeloupe, 2018, 6 p.

Olier, Nicole, « Tourisme et environnement au niveau du Parc national de Guadeloupe », in JeanMarie Breton (dir.), Tourisme, environnement et aires protégées (Antilles, Guyane, Haïti, Québec), Ed. Karthala, 2004, p. 407-431.

Parc national de la Guadeloupe, Schéma directeur d'aménagement du Parc national de la Guadeloupe, Parc national de la Guadeloupe et DAT Conseils, 1997, 118 p.

Romelaer, Pierre, «L'entretien de recherche », in Patrice Roussel et Frédéric Wacheux, Management des ressources humaines : méthodes de recherche en sciences humaines et sociales, De Boeck, 2005, p. 101-137.

Vaslet, Amandine, Chevry, Laury, Allonde, Neil, et Brugneaux, Sophie, Analyse régionale Guadeloupe - Synthèse des connaissances, Université des Antilles et de la Guyane, Parc national de Guadeloupe, Agence des Aires marines protégées, 2013, 268 p.

Warichi, La Baie du Grand Cul-de-Sac Marin : éléments pour un schéma d'interprétation, 2001, 68 p.

\section{NOTES}

1. Au milieu des années 1990 , le Parc national estime la fréquentation touristique des chutes du Carbet à 500000 visiteurs/an, de la cascade aux Écrevisses sur la route de la traversée à 500000 visiteurs/an et du volcan de la Soufrière à 250000 visiteurs/an (Parc national de la Guadeloupe, 1997). 
2. Le GCSM renferme $60 \%$ des espèces de Gorgones et la majorité des espèces coralliennes des Antilles françaises.

3. Les nouvelles clientèles (touristes en provenance d'Amérique du Nord, de la Martinique...) semblent particulièrement intéressées par l'offre alternative de tourisme de nature et de sports d'eau (Observatoire régional du Tourisme, 2018).

4. Il s'agit souvent d'une saintoise, barque de pêche reconvertie pour le transport de passagers et munie d'un à deux moteurs hors-bord.

5. La catégorie des «touristes" intègre à la fois les «vrais » touristes et les touristes originaires de la Guadeloupe mais vivant en métropole Parmi eux, une moitié a déclaré venir pour la première fois dans le lagon, tandis que l'autre moitié a déclaré des parcours similaires aux parcours des touristes primo-visiteurs.

6. Les Mercury Days sont des journées festives organisées à l'îlet Caret entre 2005 et 2007. Elles ont rassemblé plus de 700 bateaux et plus de 3000 personnes d'un coup.

7. Le montant de la Taxe Barnier est fixé à $7 \%$ du prix hors taxes du titre aller et à $3,5 \%$ du titre aller-retour. Un plafond est fixé à 1,65 euros par passagers.

\section{RÉSUMÉS}

La conciliation entre conservation patrimoniale et ouverture au tourisme est une question clé dans le Grand Cul-de-Sac Marin en Guadeloupe. Ce dernier est à la fois un espace exploité pour ses ressources marines, une destination de loisirs pour les populations locales, et un espace touristique sur lequel s'est structuré tout un secteur de prestations de tourisme vendues à l'échelle de l'archipel. Cette situation qui explique l'invention d'un haut-lieu touristique et patrimonial n'est pas sans poser un certain nombre de difficultés. La grande concentration des pratiques touristiques dans les espaces de plus forte sensibilité et valeur écologiques nécessite ainsi de trouver des solutions innovantes de gestion. La gouvernance de la baie fait également l'objet d'une refonte importante dans laquelle le Parc national est invité à se défaire de son rôle de "gendarme" pour proposer une concertation accrue avec les usagers. Même si tous les acteurs locaux (habitants, touristes, prestataires touristiques, collectivités locales) n'ont pas la même vision de la place à laisser aux usages touristiques, les trois visions qu'ils développent aujourd'hui au sujet de l'espace protégé témoignent néanmoins d'une appropriation patrimoniale bien partagée mais aussi d'un grand attachement aux îlets et aux milieux naturels. Le débat est donc celui du degré de protection et des moyens de gestion à choisir pour limiter les impacts environnementaux du tourisme.

The balance between heritage conservation and tourism development is a key issue in the Grand Cul-de-Sac Marin in Guadeloupe. The lagoon was recently invented as a touristic and heritage site and cumulates three functions: i) it's an area exploited for its marine resources, ii) it's a leisure destination for the local community, and iii) it's a tourist destination where a tourism services sector has been developed throughout the archipelago. This context leads to specific difficulties and issues. The great spatial concentration of tourism practices in areas of greater sensitivity and ecological value requires finding innovative management solutions. The governance of the bay is also the subject of a major overhaul in which the National Park is invited to shed its role of "gendarme" to propose increased consultation with users. Even if all the local stakeholders (inhabitants, tourists, tourist services providers, local communities) do not have the 
same vision of the place to leave to touristic development, the three visions they have today about the protected area demonstrate that they share the same heritage values and the same attachment to the islets and the natural environment. The debate is therefore about the degree of protection and the management tools which can limit the environmental impacts of tourism.

La reconciliación entre la conservación del patrimonio y la apertura al turismo es un tema clave en el Grand Cul-de-Sac Marin en Guadalupe. La bahía es una zona explotada por sus recursos marinos, un destino de ocio para las poblaciones locales y una zona turística en la que se ha estructurado todo un sector de servicios turísticos vendidos en todo el archipiélago.

Esta situación, que explica la invención de un sitio turístico y patrimonial, plantea una serie de dificultades. La gran concentración de prácticas turísticas en áreas de mayor sensibilidad y valor ecológico requiere, por lo tanto, la búsqueda de soluciones de gestión innovadoras. La gobernanza de la bahía también es objeto de una importante revisión en la que se invita el Parque Nacional a abandonar su papel de "gendarme" para proponer una mayor consulta con los usuarios. Incluso si todos los actores locales (habitantes, turistas, proveedores de servicios turísticos, comunidades locales) no tienen la misma visión del lugar para irse a los usos turísticos, las tres visiones que desarrollan hoy sobre el espacio protegido demuestran que tienen la misma visión del patrimonio y el mismo apego a los islotes y el ambiente natural. Por lo tanto, el debate es sobre el grado de protección y los medios de gestión para limitar los impactos ambientales del turismo.

\section{INDEX}

Keywords : Marine Protected Area, National Park, tourist flows, management, climate change, tropical island, lagoon, Lesser Antilles, Guadeloupe

Mots-clés : aire marine protégée, Parc national, fréquentation touristique, gestion, changement climatique, île tropicale, lagon, Petites Antilles, Guadeloupe

Palabras claves : Área marina protegida, Parque nacional, turismo, manejo, cambio climático, isla tropical, laguna, Antillas menores, Guadalupe

\section{AUTEURS}

\section{VINCENT ANDREU-BOUSSUT}

Maître de conférences en géographie. UMR ESO 6590 CNRS, Le Mans Université. vincent.andreuboussut@univ-lemans.fr

\section{CAROLINE RUFIN-SOLER}

Maître de conférences en géographie. TVES EA 4477, Université du Littoral Côte d'Opale. caroline.rufin-soler@univ-littoral.fr

\section{CÉLINE CHADENAS}

Maître de conférences en géographie. UMR LETG 6554 CNRS, Université de Nantes. celine.chadenas@univ-nantes.fr 